\title{
Trailing Exophthalmos Revealing Neurofibromatosis: In A Case Report and Review of the Literature
}

\author{
B. Zouita*, B. Bannar, M.Ranib, D. Basraoui, H. Jalal
}

Radiology service mother and child hospital CHU Mohammed VI Faculty of Medicine and Pharmacy of Marrakech Morocco

DOI: $10.36347 /$ sjmcr.2021.v09i04.019

| Received: 26.02.2021 | Accepted: 30.03.2021 | Published: 22.04.2021

*Corresponding author: B. Zouita

Abstract

Case Report

Neurofibromatosis 1 is the most common phacomatosis and neurofibromatosis, with an incidence of $1 / 2500$ births. Transmitted in the autosomal dominant mode, it results in the presence of tumors mainly of the skin, cerebral and peripheral nerves, and in bone and vascular dysplasias. In this presentation, we report the case of a 4-year-old girl, who had consulted for a trailing exophthalmos for two years without any particular pathological history, but the scanner objected to sphenoidal dysplasia with right hemi-megalencephaly, in front of the worsening of her exophthalmos, an MRI was performed with clinical reassessment which demonstrated the appearance of café-au-lait spots with OBNIs and a plexiform neurofibroma on MRI.

Keywords: Exophthalmos, CT, MRI, neurofibromatosis.

Copyright $\left({ }_{0} 2021\right.$ The Author(s): This is an open-access article distributed under the terms of the Creative Commons Attribution 4.0 International License (CC BY-NC 4.0) which permits unrestricted use, distribution, and reproduction in any medium for non-commercial use provided the original author and source are credited.

\section{INTRODUCTION}

Neurofibromatosis type 1 (NF1), formerly known as von Recklinghausen's disease is the most common phacomatosis, a group of diseases characterized by the association of tumors of the skin and the nervous system [1].

The disease incidence is $1 / 2500$ births and the prevalence is $1 / 4000$ to $1 / 5000$, with no gender predominance [2]. NF1 is a progressive multisystem disease which mainly affects the skin, eyes, central and peripheral nervous system, skeleton and vessels. The lesions observed may be tumors, hamartomas or dysplasias.

\section{Clinical Observation}

We report the case of a 4-year-old girl, followed for a trailing exophthalmos, without abnormalities on clinical examination, the patient underwent a first CT scan showing sphenoidal dysplasia with right hemi-megalencephaly, during the Follow-up of the patient the exophthalmos was worsened and an MRI was requested with clinical reassessment which demonstrated the appearance of café au lait spots with OBNI and plexiform neurofibroma on MRI.

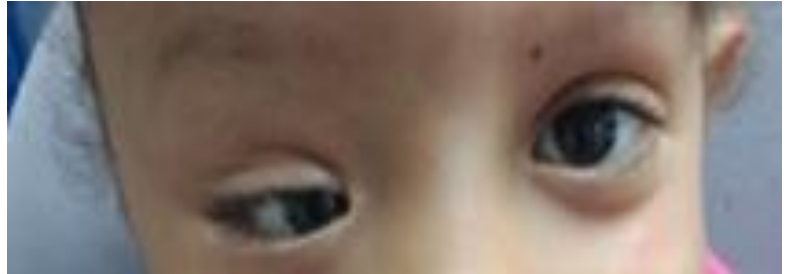

Fig-1: Right exophthalmos

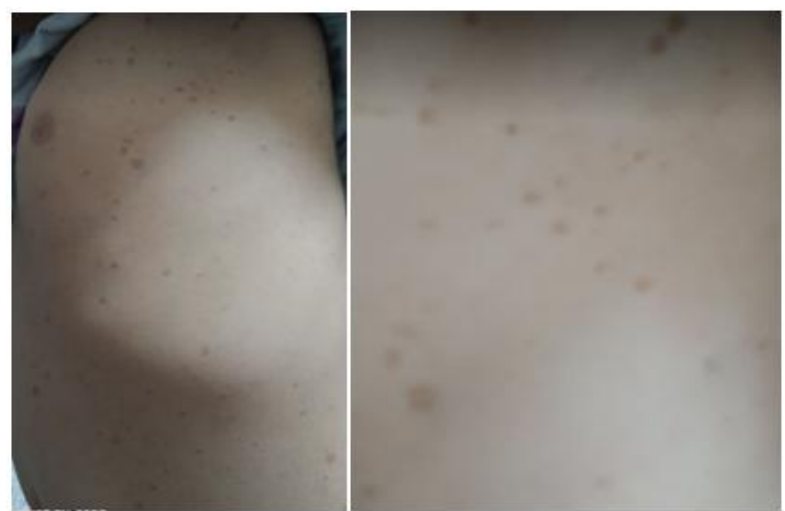

Fig-2: Cafe au lait stains skin 

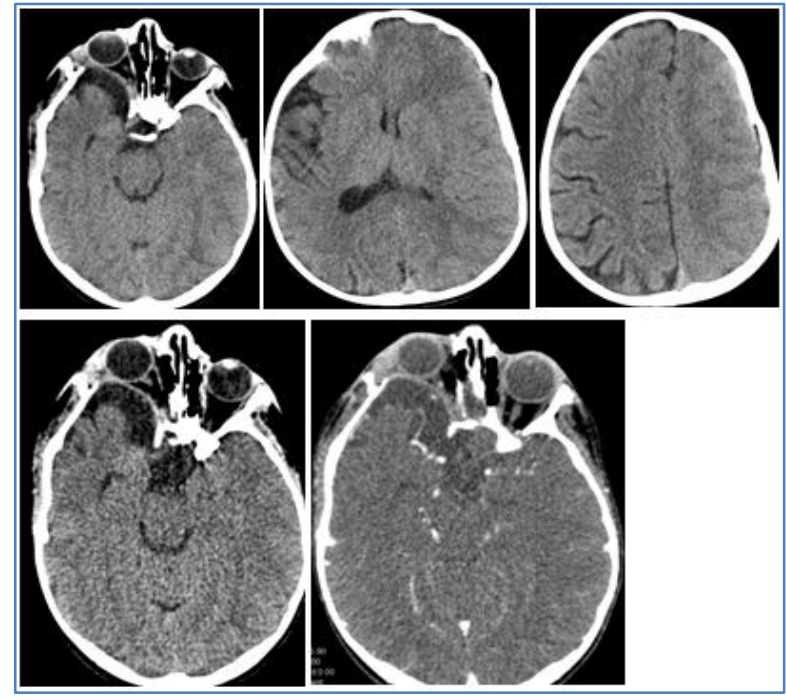

Fig-3.a

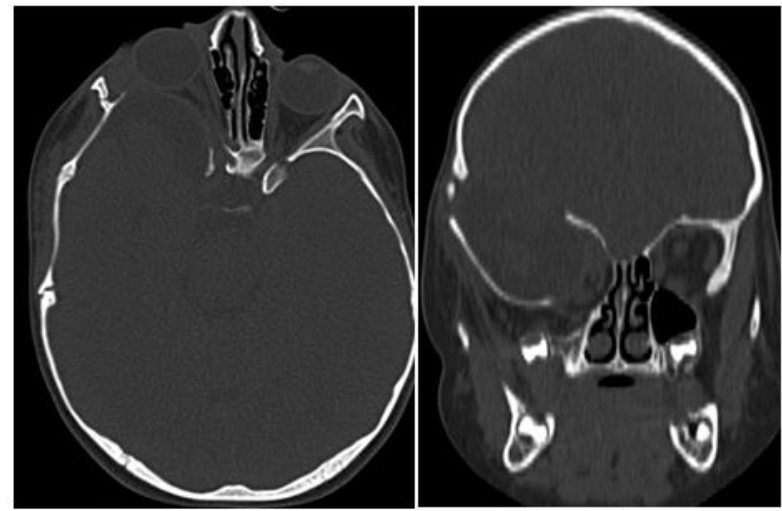

Fig.3.b

CT in the parenchymal window (fig.3.a) and in the bone window (fig.3.b) deformation with right spheno-orbital dysplasia and dilation of the subarachnoid spaces opposite, producing the appearance of a hemi-megalencephaly.

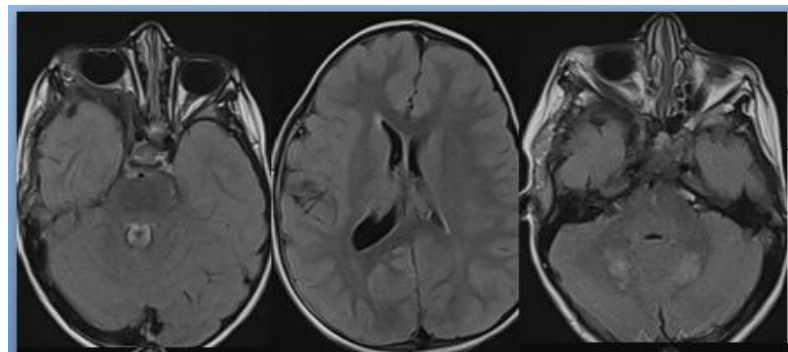

FLAIR MRI Sequence

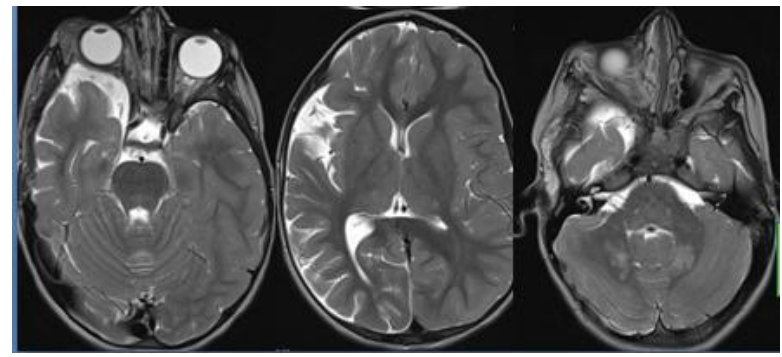

T2 SE MRI Sequence

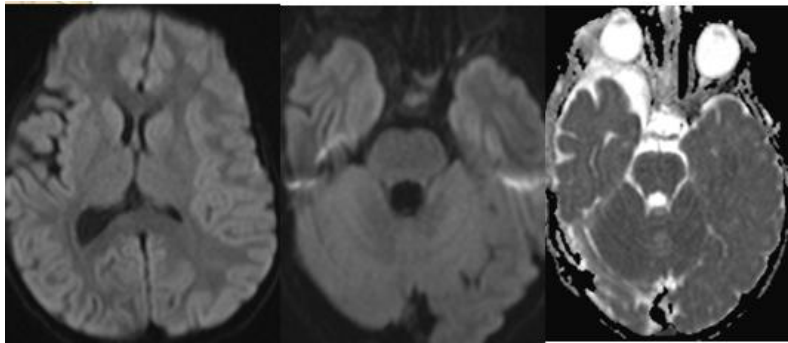

DWI MRI Sequence

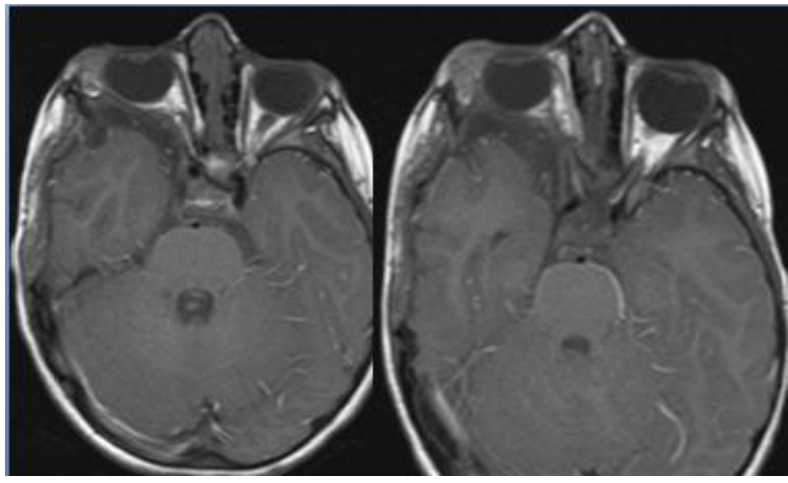

T1 SE MRI Sequence

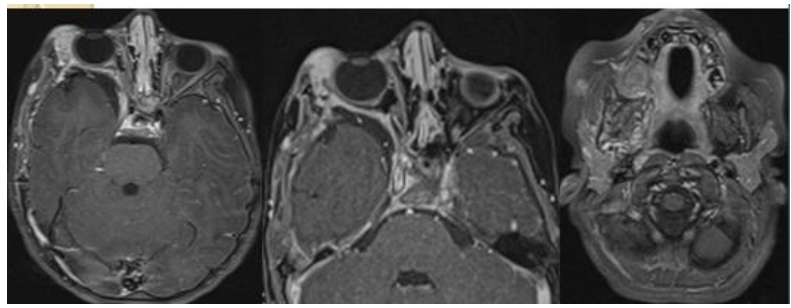

MR imaging by 3D T1-weighted post gadolinium injection

Fig-4: Sphenoidal dysplasia with dilation of the subarachnoid spaces associated with meningeal thickening of the soft temporal subcutaneous parts. Swollen appearance of the lacrimal gland in relation to an intraglandular plexiform neurofibroma, the whole is responsible for a grade I exophthalmos. Signal abnormalities at the level of the cerebellar hemispheres related to Unidentified Shiny Objects

\section{DISCUSSION}

Neurofibromatosis is a protean disease that can affect most organs. Imaging is used at the diagnostic stage, and in the early detection and follow-up of complications. Knowledge of the panel of pathologies associated with NF1 is essential in order to adapt the imaging protocol to the organ being explored and to the patient's age [1].

Imaging may suggest the diagnosis of NF1 in the presence of characteristic lesions. It is mainly involved in the detection and monitoring of complications of the disease. The lesions are visible on imaging mainly in the brain, spine, peripheral nervous system and long bones.

Glioma of the optic pathways GVO is the most common brain tumor in children with NF1, observed in 
$15 \%$ of cases [2]. It occurs before the age of 7 years, with a peak incidence of 4 to 5 years, but can occur later and in adults [3].

The tumor causes an enlargement and a tortuous aspect of the optic nerves, which appear isointense in T1, hypo- or hyper-intense in T2, with an effacement of the peri-nervous subarachnoid spaces. The contrast enhancement is variable, observed in $56 \%$ of cases [4]. We can only observe tumor infiltration of the meningeal sheath and the subarachnoid spaces around the optic nerve, enhanced after injection of gadolinium, with an intact optic nerve [5]. This is to be distinguished from a simple ectasia of the meningeal sheath of the optic nerve by dural dysplasia [6], without associated contrast enhancement.

Hamartomas, or Unidentified Shiny Objects (OBNI), are benign brain lesions very commonly seen in NF1 patients. These lesions are found in the thalamostrieal regions $(75-80 \%)$ [7], the cerebellar white matter, the brainstem (bridge, midbrain) and the splenium of the corpus callosum. They are often found during MRI scans for a GVO.

The dysplasia or dural ectasia in NF1 is classically attributed to a primary mesodermal dysplasia of the meninges. It results in an expansion of the meninges under the effect of pulsations of cerebrospinal fluid, which causes indentations on the posterior surface of the vertebral bodies (scalloping).

Neurofibromas are observed in $40 \%$ of cases [8], they are rare intradural, and most often located along the roots of the cauda equina. They are more often foraminal, where they widen the foramina, and multiple paraspinal. Few symptomatic (2\%), especially in children, they can cause spinal cord compression and are often associated with scoliosis [9]. In the sporadic and familial forms of spinal neurofibromatosis, multiple bilateral spinal neurofibromas are observed.

There is also Plexiform neurofibromas are tumor invasion of a long portion of a nerve and its branches, or of a nerve plexus. Macroscopically, they present a multi-lobulated appearance in a cluster, string or bag of worms, or sometimes the appearance of an irregular mass more diffuse and infiltrating [10].

Sometimes we can objectify lesions or bone malformations such as scoliosis or dysplasia of the large sphenoid wing.

\section{Conclusion}

NF1 is a progressive disease, but its course is impossible to predict in any given patient. This involves regular multidisciplinary follow-up in a referral center in order to detect early on the most serious complications of the disease, which depend on the patient's age.

\section{REFERENCES}

1. Kooba JL. Dietemann Imagerie de la neurofibromatose de type $1 \S$ Neurofibromatosis type 1 imaging $\mathrm{M}$.

2. Ferner RE. The neurofibromatoses. Pract Neurol. 2010;10:82-93.

3. Cecen E, Ince D, Uysal KM, Ozer E, Cetingoz R, Ozguven AA. Soft tissue sarcomas and central nervous system tumors in children with neurofibromatosis type 1 . Childs Nerv Syst. 2011;27:1885-93.

4. Guillamo JS, Creange A, Kalifa C, Grill J, Rodriguez D, Doz F. Prognostic factors of CNS tumours in Neurofibromatosis 1 (NF1): a retrospective study of 104 patients. Brain. 2003; 126(Pt1):152-60.

5. Chung EM, Specht CS, Schroeder JW. From the archives of the AFIP: Pediatric orbit tumors and tumorlike lesions: neuroepithelial lesions of the ocular globe and optic nerve. Radiographics. 2007; 27:1159-86.

6. Barkovich WK. Pediatric Neuroimaging. Philadelphia: Lippincott Williams and Wilkins; 2011.

7. Barbier C, Chabernaud C, Barantin L, Bertrand P, Sembely C, Sirinelli D. spectroscopic imaging of basal ganglia and thalamus in neurofibromatosis type 1: correlation with T2 hyperintensities. Neuroradiology. 2011; 53:141-8.

8. Pascual-Castroviejo I, Pascual-Pascual SI, Viano J, VelazquezFragua R, Lopez-Gutierrez JC. Bilateral spinal neurofibromas in patients with neurofibromatosis 1. Brain Dev. 2012; 34: 563-9.

9. Barkovich WK. Pediatric Neuroimaging. Philadelphia: Lippincott Williams and Wilkins; 2011.

10. Woodruff JM. Pathology of tumors of the peripheral nerve sheath in type 1 neurofibromatosis. Am J Med Genet. 1999; 89: 2330 . 